\title{
Research on General Programming Environment Technology Based on Web
}

\author{
Liu $\mathrm{Yu}^{1, \mathrm{a}}$ \\ ${ }^{1}$ School of Software Technology, Zhejiang University, Zhejiang, China
}

\begin{abstract}
As the MOOC develops, many users have begun to gradually like and get used to programming learning on the MOOC platform, so virtual online experiments have become a hot spot in the online programming education industry, which is also known as online IDE or online programming environment. Compared with the traditional programming environment, the online programming environment does not require a complicated establishment of local environment, making it easy to be used. At the same time, the online programming environment can also be integrated into the MOOC for learning, and can also be combined with $\mathrm{OJ}$ for evaluation. However, the existing online programming environment usually only supports few programming languages, such as $\mathrm{C}$ language and Java language. Even some online platforms only have the online compiling function with one language. Based on the above phenomena, this paper will propose a general online programming solution. And based on the idea, a platform that supports multilanguage online compiling function is built through simple code writing and the construction of server environment..
\end{abstract}

\section{Introduction}

As the Internet technology develops, online programming websites are coming into view. People can write and learn code anytime and anywhere with online programming sites. For programming beginners, the programs they write are simple in structure and logic, but they need to install software that takes up a lot of memory on their own computers, and configure a very complex development environment. Many people will encounter a lot of troubles in this process, and over time, it will reduce people's enthusiasm for learning programming. As a lightweight level, online programming platform that can meet the users' needs for code writing anytime and anywhere is especially important. Meanwhile, this way also allows beginners to attach more attention to the programming itself ${ }^{[1]}$.

At present, there are many kinds of online programming platform. Although some online programming platforms support the online writing and running with dozens of programming languages, more platforms only support the online writing and running with one or several programming languages. And these platforms are not perfect in terms of syntax highlighting, image output, automatic completion of code, and associating input. Taking the Matlab programming language as an example, there are only a handful of platforms that support the online writing and compiling of this language at home and abroad, and the platforms that support its image output are remote. Therefore, a general programming environment technology based on the Web is proposed in this paper. According to the call to $\mathrm{CMD}$ instruction and the combination of $\mathrm{ACE}$ framework, based on realizing the common functions of other online programming platforms, the syntax highlighting, image output and automatic completion of code, and associating input, standard input and parameter input, and the Matlab language image output are improved. Meanwhile, the common web development language can be used to achieve the online writing and compiling functions of most programming languages. This technology can quickly help enterprises and schools to establish their own online evaluation and test platform, and can also help online programming learning platforms or websites to quickly realize the online programming functions with high performance and good user experience, which is of great significance $[2][3[4][5]$.

\section{System background}

In recent years, the online programming develops rapidly. There are many researches and practice results about online programming systems in foreign countries, such as the CodePad, Ideone, CodeRun and Codecademy with high utilization rate. Users can write code online on these platforms, then compile and run the code, and get feedback results. Even users can open the web page through the mobile phone and write the code in some special time, so as to verify some ideas. Due to the convenience of online programming and the convenience of multi-terminal, Codecademy has obtained 100,000 registered users within 48 hours after the release. However, these websites and platforms are not ideal for

$\overline{{ }^{a} \text { Corresponding author: service@52exe.cn }}$ 
syntax highlighting and standard input, parameter input, automatic completion, and association input. They also do not support the Matlab online programming and image output functions ${ }^{[6][7]}$.

There are many researches on online programming in China, such as IMOOC, Shiyanlou and other platforms, they all have online programming functions, but the types of programming languages they support are relatively few. They are also not involved in automatic completion of code and association input, as well as the graphical output of some programs ${ }^{[8]}$.

\section{SYSTEM DEVELOPMENT}

\subsection{System structure}

The design of this system mainly provides the convenience for programmers, especially for the programming beginners or temporary users. It is also to propose a general method, which can quickly use more Web language to participate in development, and can also quickly add more programming languages. The basic structure is as shown below.

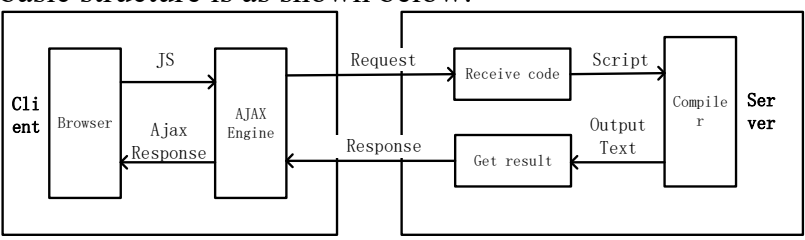

Figure 1. System Structure Diagram

According to the system structure diagram, we can roughly analyze that the system experience 7 stages in operation:

1: The client's browser initiates a request, and transmits the code standard input, and parameter input to the Ajax engine through JavaScript technology;

2: The Ajax engine in the client transmits the received data to the server through an asynchronous request;

3: The receiving code module of the server receives the data sent by the client in the POST model;

4: After receiving the data sent by the client, the server calls script to sort the received data, obtains the corresponding parameters, and passes the parameters to the script;

5: The server's script calls the source compiler on the server to run the code;

6: The result module on the server gets the result of the script run, and transmits it to the client's Ajax engine through the server response;

7: The client's Ajax engine, through the Ajax response, transmits the compiled result back to the browser and presents it.

In the whole system, the process from receiving code to call the compiler is the most important part, because this part is the core process of completing the whole project. This process is done primarily with the help of the ".sh" script document. In the script document, the compiler is called to compile the code and other information obtained by the receiving code model. It can write instructions that call different compilers together into a script, and then determine the language type by the a certain transmitted parameter, which is convenient for updating the language type in the future, and also convenient for the web development, and can be easily and quickly interfaced with our development language [9][10][11]

\subsection{Development decomposition}

According to the analysis of the system architecture, the system consists of the following three parts, the front end part, the back end part and the request part.

The implementation method of the front end part is mainly based on the ACE framework. ACE is a code editor based on Web that implements syntax coloring. The supported languages include: SQL, Ruby, SASS, PHP, Csharp, Java, etc., and the number of programming languages exceeds 40 kinds. At the same time, it also has real-time error correction function of HTML, PHP, Javascript. The ACE code framework of the latest version also has functions such as association input and automatic completion, and code folding. By combining it with the ACE framework, the front end can greatly enhance the user experience.

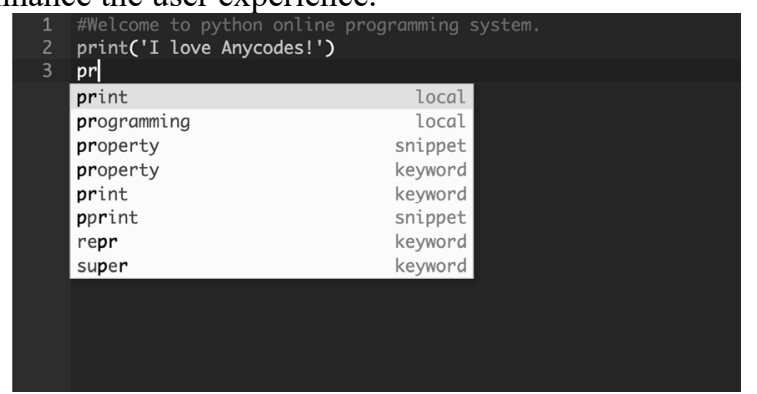

Figure 2. Front End Style by Combining ACE Frame

The back end part is mainly the calling method of the program and the content of the script. This part is also the core of the whole system. The first is to develop the calling of language to scripts. There are different calling methods for different languages. For example, the calling can be carried out by subprocess.Popen() in Python, and by system() in PHP. For the compiling of script file, taking the Ubuntu server as an example, firstly ensure that the computer can run shell script file normally, secondly that the target compiled language has properly configured the environment in the server, and then can run the code through the compiler instructions. Taking Matlab as an example: it may be abcdef, the main function of this code is to set the timeout time, and call the Matlab compiler to write the code, and several parameters of -nodesktop, -nosplash, and -nodisplay should be noted. The basic meaning is to let the Matlab compiler not compile the document (variable \$source file 2) while not displaying it ${ }^{[12][13]}$.

The request part is actually the data transmission. In this part, we mainly use the Ajax technology. It is necessary to pay special attention to that the synchronous loading can not be used in order to avoid users to refresh the entire page, and avoid the problem of increasing the server burden caused by refreshing the page or the loss 
of user code, and the asynchronous loading should be used..

\subsection{Core code demonstration}

Running $\mathrm{C}$ language is taken as an example, and the core running code is: timeout 10 gcc \$source_file -I . -o \$app $-\operatorname{lm}>\&$ \$debug log, the code just sets the timeout and compiles the code through gcc, where \$source file is the incoming code. When the program runs successfully through the compilation of gcc, it needs to run the corresponding substitution parameters: timeout 3 echo "\$in_flag" | timeout 3 .\$app 'echo \$7', which mainly sets the timeout and parameters or standard input.

And taking the Python language as an example, it can be written as "\$in_flag" | timeout 3 python \$source_file 'echo $\$ 7^{`}>\&$ \&debug_log, which means that the timeout is set as time, and the code is run, which supports standard input.

After comparing the background processing methods of Python and $\mathrm{C}$ language, the so-called general programming environment refers to all the processing can be placed in the shell by calling the different compiled and running instructions with different languages under the Ubuntu system and getting feedback. Then it can be called directly through the Web programming language, and transfer parameters. Thus the basic structure of the system has been built, then we will test it below.

\section{System test}

In order to test the function and performance of the system, we separately test those of the system.

System function detection. Through the writing and running of the code, it can be found that the user side (client) of the system can achieve syntax highlighting, association input, automatic completion of code, and image output, standard input and other functions. The system are very similar with local compiler in terms of code input, which is in line with most programmer programming habits.

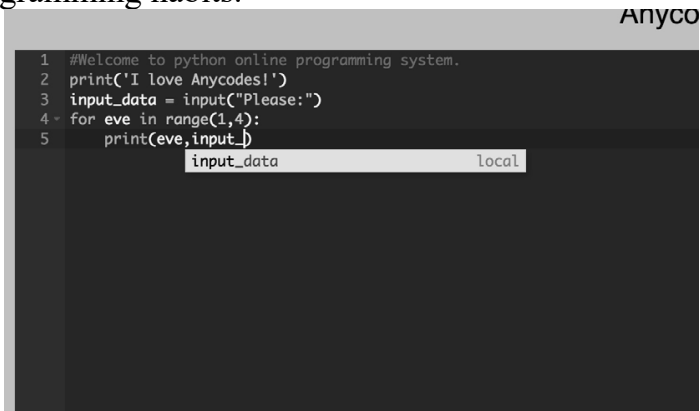

Figure 3. Code Highlighting, Code Folding, and Association Input Functions

Meanwhile, through the test and run of languages, such as abcd, efg and hijk, it is found that the output results are consistent with the output results of the local compiler.

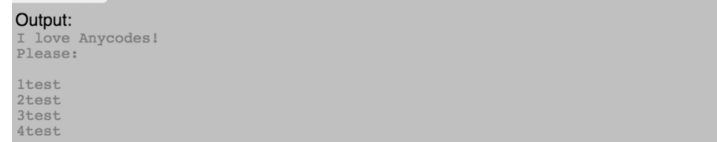

Figure 4. Python3 Standard Input and Code Run Test

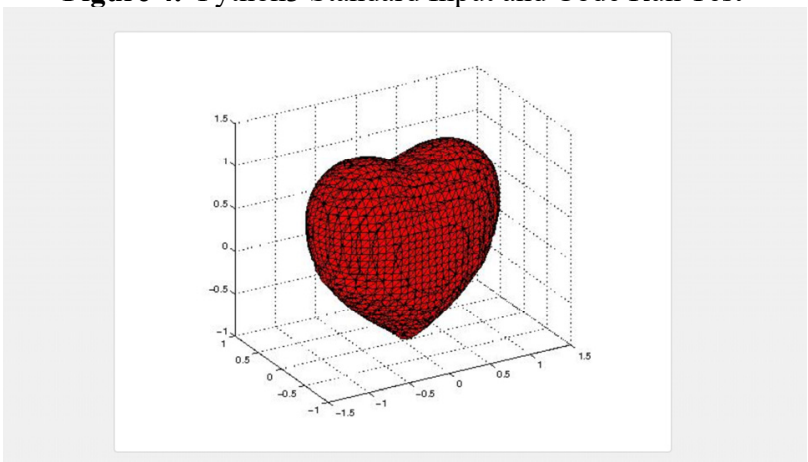

Figure 5. Matlab Image Output Test

It can be seen that the user experience of this system is good and the output results are accurate, proving that the system is feasible.

Requests are continuously sent to the server to test the performance of the system, and under the premise of smooth broadband guarantee, take the start of sending as time node and getting the output results as the end. Continuously send 100 times of requests to the nondrawing program. The abscissa axis in the table indicates the time interval (in $\mathrm{ms}$ ), and the vertical axis indicates the times of complete requests completed during that interval:

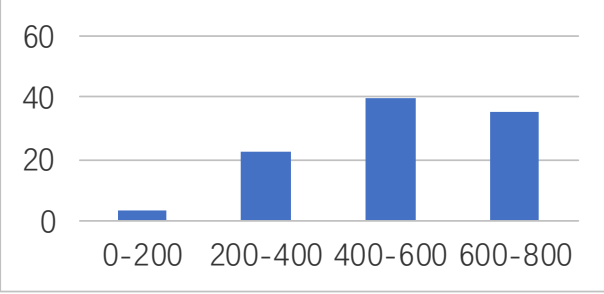

Figure 6. System Stress Test (taking Python as an example)

In the same operation condition, continuously send 100 requests to the drawing program. The abscissa axis represents the time interval (in s), and the vertical axis represents the times of complete requests completed during that interval:

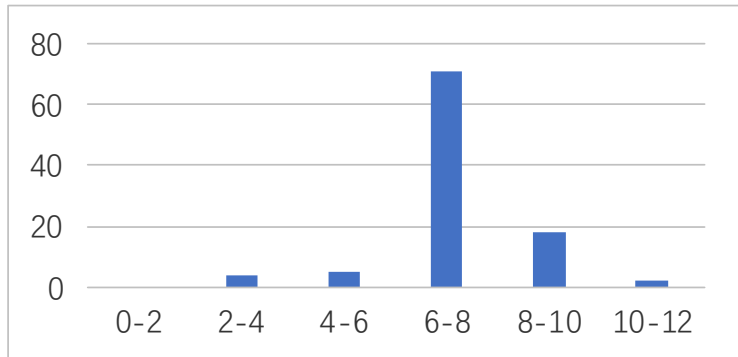

Figure 7. System Stress Test (taking Matlab drawing function as an example)

It can be found from the system test results that, when the non-drawing program is running, taking Python code as an example and running 100 times, complete a complete compilation from $200 \mathrm{~ms}$ to $600 \mathrm{~ms}$, which proves that the system has good fluency. It does not catch up during the test, and the interval between request and feedback is rapid, which proves that the 
system performed well. Although in the case of Matlab $3 \mathrm{D}$ drawing, the time becomes $6 \mathrm{~s}$ to $8 \mathrm{~s}$, but the test results are correct, and there is no error in results or incomplete image, which proves the feasibility of the system again. It should also be noted that, compared to the general program, when the Matlab drawing program runs in the local compiler, the the specific time is relatively long.

In summary, the system performs well and is feasible during the user experience and performance testing process of the system.

\section{Summary}

A multi-language online compilation platform based on WEB technology is built, and the programmer's installation of each language compilation environment is also simplified in this paper through the proposed online programming idea. Compared with other online programming platforms, this compilation platform implements image output function, association input function and parameter input function. The core function of online programming is written as a script. Through the receiving code module, the user code is received and the script is called. Then the user's code is compiled through the instructions in the script that compiles the language, and the feedback is obtain and transmitted to the user. Thus the online programming is achieved. The above practice fully proves the feasibility of this view. Based on this point of view, we can build other online platforms, such as online evaluation, online exam, and so on. laying the foundation for the future development of online systems, and protecting the future development of WEB technology.

\section{References}

1. Chen Liang. Web-Based C Language Source Code Level and Assembly Level Debugger [D]. University of Electronic Science and technology of China, 2016.

2. Wu Jianfei, Wu Liang, Zhang Shuzhen. Design and Implementation of Online Evaluation System [J]. Software Guide, 2009(1): 77-78.

3. He Zhaoqing. ASP Technology Based on Web Network Programming[J]. Journal of Shaoyang University: Social Sciences, 2002, 1(6): 74-77.

4. Ma Shihuan, Zhang Jin. Research on Web-based Programming Teaching[J]. Curriculum Education Research: New Teacher Teaching, 2013(9).

5. Liu Zhikai, Zhang Taihong, Liu Lei, et al. Webbased Python3 Programming Environment[J]. Computer Systems and Application, 2015, 24(7): 236-239.

6. Parnin C, Rugaber S. CodePad:interactive spaces for maintaining concentration in programming environments[C]// Softvis '10: Proceedings of the, International Symposium on Software Visualization. 2010:15-24.
7. Anonymous. CodeRun: Cloud Programming Development IDE of Browser [J]. Silicon Valley, 2011(7):45-45.

8. Ge Xiangwei. Research on the Cultivation of Programming Style Based on WEB [D]. Inner Mongolia Normal University, 2013.

9. Qin Yuelei, Peng Siwei. Design and Implementation of Source Code Online Evaluation System[J]. Fujian Computer, 2008, 24(6): 156-156.

10. Ji Hongbo. Design and Implementation of Online Evaluation System of Web Source Program Based on jQuery [D]. Jilin University, 2010.

11. Cai Chongchao. Design and Implementation of Online Judgment System Based on $\mathrm{Web}[\mathrm{J}]$. Software Guide, 2016, 15(3): 107-109.

12. Liu Zhikai. Research on Python Programming Environment Based on Web[D]. Xinjiang Agricultural University, 2015.

13. Liu Kai, Wang Lumei. Improving the Quality of $\mathrm{C}$ Language Teaching by OnlineJudge[J]. Modern Computer Monthly, 2012(10):48-50. 